\title{
The homeodomain-containing transcription factor, GhHOX3, is a key regulator of cotton fiber elongation
}

\author{
PEI Yan \\ Biotechnology Research Center, Southwest University, Chongqing 400716, China \\ Received January 13, 2015; accepted January 16, 2015; published online January 27, 2015
}

Citation: $\quad$ Pei Y. The homeodomain-containing transcription factor, GhHOX3, is a key regulator of cotton fiber elongation. Sci China Life Sci, 2015, 58: 309-310, doi: 10.1007/s11427-015-4813-8

Recently, Chen and colleagues from Shanghai Institute of Plant Physiology and Ecology of Chinese Academy of Sciences, in collaboration with scientists from Nanjing Agricultural University and from the US, have identified a homeodomain (HOX)-containing transcription factor that plays a central role in controlling cotton fiber elongation [1].

Cotton is widely cultivated in over 80 countries mainly for cotton fiber, the most important natural and renewable raw material for textile industry. Cotton fibers are single-celled trichomes, and their development can be divided into four overlapping stages including initiation, elongation, secondary cell wall biosynthesis and maturation. Cell elongation largely determines fiber length, a key quality and yield trait. In addition, the extensively elongated fiber cells, which undergo semi-synchronized development and rapid growth, provide an excellent system for the study of plant cell elongation. Recent investigations have shown that a MIXTA-type MYB transcription factor, GhMYB25-like, acts as a major regulator of fiber initiation [2], and a bi-functional LIM domain protein, WLIM1a, is involved in fiber cell wall biosynthesis and possibly also elongation [3]. However, how fiber elongation is controlled remains largely unknown.

Based on genetic analysis, Shan et al. [1] discovered that GhHOX3 gene, which encoded an HD-ZIP IV transcription factor, associated with fiber quality traits, particularly the fiber length. Silencing of GhHOX3 gene resulted in a dra-

email: peiyan3@swu.edu.cn matic decrease of fiber length ( $>80 \%$ decrease, lint completely disappeared), while fiber initiation was largely unaffected. Furthermore, overexpression of GhHOX3 gene promoted fiber elongation. They identified 78 downregulated genes in the suppression line and most of which were classified into biological processes of cell growth, including cell wall formation, transcriptional regulation, signal transduction and metabolism. Of these, two cell wall loosening genes, GhRDLl and GhEXPAl, were direct targets of GhHOX3, as evidenced both in vitro and in planta. GhHOX3 was further proved to directly bind to the L1 box cis-element present in the promoter of both GhRDL1 and GhEXPA1.

Phytohormones, including auxin, gibberellin (GA) and probably ethylene, are proposed to regulate cotton fiber initiation and elongation [4,5]. Spatiotemporally 'fine-tuning' auxin biosynthesis in cotton ovule epidermal cells promotes fiber initiation, thus results in significant enhancement of fiber yield and quality [4]. However, the molecular mechanism underlying the hormone-mediated fiber cell elongation remains elusive. When culturing cotton ovules, GA is indispensable for fiber cell growth. In addition, GA can up-regulate the expression of $G h R D L 1$ and GhEXPAl genes, and this induction requires GhHOX3. These data not only indicate an essential role of GA in fiber elongation but also suggest the functional involvement of HOX proteins in GA signaling pathway. To dissect the GA-mediated regulatory network, the authors employed yeast two-hybrid screening for proteins interacting with GhHOX3. Multiple transcription factors involved in phyto- 
hormone function and plant development were isolated, including another HD-ZIP IV protein, GhHD1, that was reported to have a mild effect on fiber cell development, and GhSLR1, a DELLA protein acting as major repressor of GA signaling. GhSLR1 did not bind to GhHD1, but competed for the interaction with GhHOX3. Such triple-protein-interaction indeed affected the transcriptional activity of downstream genes: the GhHOX3-GhHD1 complex synergistically activated gene expression, and thus activity was substantially repressed by GhSLR1.

This research uncovers the molecular mechanism of GA

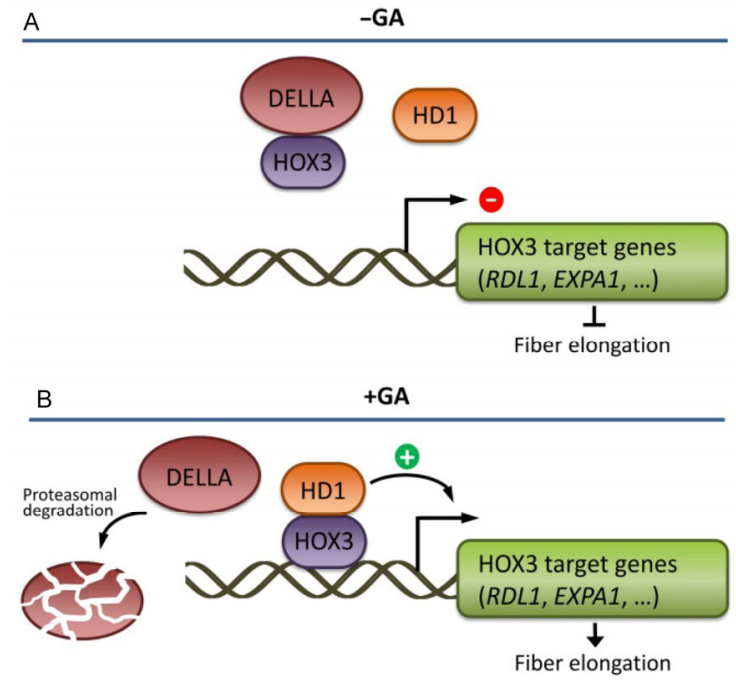

Figure 1 (color online) A model for regulation of cotton fiber elongation by GhHOX3 and the phytohormone GA [1]. A, In cotton fibers, DELLA proteins bind to HOX3 to prevent its binding to other HD-ZIP transcription factors, repressing their transcriptional activation to target genes. B, GAs trigger degradation of DELLAs, releasing HOX3 protein to interact with other HD proteins, such as HD1, allowing activation of target genes, including $R D L 1$ and EXPA1, to promote cotton fiber elongation. in promoting cotton fiber elongation, in which GhHOX3 is a core regulator, and other regulatory proteins, such as GhHD1, act as accessory factors in the regulatory complex. DELLA proteins prevent the formation of the active complex through directly binding to GhHOX3, and the high level of GA triggers degradation of DELLA proteins, releasing GhHOX3 to interact with other transcription factors, allowing the activation of target genes and the resultant cotton fiber elongation (Figure 1). This HOX protein-involved GA signaling pathway may work in other types of plant cell rather than unique to cotton fiber. Further characterization of the regulatory network centering on GhHOX3 will help to identify other important regulators of cotton fiber development, and will definitely provide precious information and candidate genes for improving cotton fiber quality and yield through molecular breeding.

1 Shan CM, Shangguan XX, Zhao B, Zhang XF, Chao L, Yang CQ, Wang LJ, Zhu HY, Zeng YD, Guo WZ, Zhou BL, Hu GJ, Guan XY, Chen ZJ, Wendel JF, Zhang TZ, Chen XY. Control of cotton fibre elongation by a homeodomain transcription factor GhHOX3. Nat Commun, 2014, 5: 5519

2 Walford SA, Wu YR, Llewellyn DJ, Dennis ES. GhMYB25-like: a key factor in early cotton fibre development. Plant J, 2011, 65: 785-797

3 Han LB, Li YB, Wang HY, Wu XM, Li CL, Luo M, Wu SJ, Kong ZS, Pei Y, Jiao GL, Xia GX. The dual functions of WLIM1a in cell elongation and secondary wall formation in developing cotton fibers. Plant Cell, 2013, 25: 4421-4438

4 Zhang M, Zheng X, Song S, Zeng Q, Hou L, Li D, Zhao J, Wei Y, Li X, Luo M, Xiao Y, Luo X, Zhang J, Xiang C, Pei Y. Spatiotemporal manipulation of auxin biosynthesis in cotton ovule epidermal cells enhances fiber yield and quality. Nat Biotechnol,2011, 29: 453-458

5 Shi YH, Zhu SW, Mao XZ, Feng JX, Qin YM, Zhang L, Cheng J, Wei LP, Wang ZY, Zhu YX. Transcriptome profiling, molecular biological, and physiological studies reveal a major role for ethylene in cotton fiber cell elongation. Plant Cell, 2006, 18: 651-664

Open Access This article is distributed under the terms of the Creative Commons Attribution License which permits any use, distribution, and reproduction in any medium, provided the original author(s) and source are credited. 